\title{
THE SPACE DENSITY OF HIGH-REDSHIFT QUASARS
}

\author{
P.A. SHAVER ${ }^{1}$, J.V. WALL ${ }^{2}$, K.I. KELLERMANN ${ }^{3}$, C. JACKSON ${ }^{4}$ \\ AND M.R. HAWKINS \\ 1 European Southern Observatory - Karl-Schwarzschild-Str. 2, \\ 85748 Garching, Germany \\ ${ }^{2}$ Royal Greenwich Observatory - Madingley Road, Cambridge \\ CB3 OEZ, England \\ 3 National Radio Astronomy Observatory - Edgemont Road, \\ Charlottesville, VA 22903, U.S.A. \\ ${ }^{4}$ Institute of Astronomy - University of Cambridge, Madingley \\ Road, CB3 OHA, England \\ ${ }^{5}$ Royal Observatory - Blackford Hill, Edinburgh EH9 $3 H J$, \\ Scotland
}

\begin{abstract}
An upper limit on the space density of quasars at $z>5$ is obtained independent of any optical magnitude limit, from the complete identification of a large sample of flat-spectrum radio sources. This upper limit is below the observed space density at $z \sim 2-3$, showing that the turnover in space density is real and not merely due to obscuration.
\end{abstract}

We are completing the optical identification of a large sample of flatspectrum radio sources, in order to search for high-redshift quasars and determine their space density. The sample is comprised of 896 flat-spectrum sources $(\alpha>-0.4$ between 2.7 and $5 \mathrm{GHz})$ from the Parkes catalogue with $S_{2.7} \gtrsim 0.25 \mathrm{Jy}$ in the range $+2.5^{\circ}>\delta>-80^{\circ}$, of which 581 were already identified in the catalogue, $83 \%$ with quasars. VLA and Australia Telescope positions were obtained for the unidentified sources, accurate to $<1$ arcsec, and UKST/COSMOS $B_{J}$ identifications were then made for 185 of these. CCD imaging observations with EFOSC on the ESO $3.6 \mathrm{~m}$ telescope in the $B$, Gunn- $i$, and Gunn- $z$ bands were made for the remaining sources.

From high-frequency observations of $z \lesssim 2$ flat-spectrum radio QSOs, it is known that the observed spectra between 2.7 and $5 \mathrm{GHz}$ would still be flat for redshifts up to 10 . It is also known that intervening Lyman- 
limit absorption completely obscures $z>5$ quasars in the optical $B$-band. Thus, in searching for $z>5$ quasars we seek flat-spectrum sources which are obscured in the $B$-band. Tentatively, it appears that all sources can be identified either with galaxies or with stellar objects which are present in the $B$-band (and therefore not at $z>5$ ). Only one very red stellar identification has been found, which was marginally present in the $B$-band (hence at high redshift, but not at $z>5$ ): PKS 1251-407. Its redshift was found to be $z=4.46$, making it the highest-redshift radio source presently known (Shaver, Wall, Kellermann 1995, MNRAS in press). Objects at higher redshifts could have been found with similar ease. If, as now seems likely, there are no unidentified sources left in the sample which could be associated with quasars at $z>5$, this gives a firm upper limit on the space density at $z>5$ which is independent of any optical magnitude limit.

Comparison of the upper limit on the space density of radio quasars at high redshift $(5<z<7)$ with the measured space density for similar objects at lower redshift gives a straightforward measure of the relative space density. A flux density limit of $0.25 \mathrm{Jy}$ was adopted, and a selection was made of similar objects $(\alpha>-0.4$, and radio power corresponding to $0.25 \mathrm{Jy}$ at $z=7$ ) at lower redshifts from the Parkes catalogue in the declination range $+10^{\circ}>\delta>-45^{\circ}$ where redshift information is $70 \%$ complete. This provided lower limits on the space density at $z<5$, to be compared with the upper limit at $5<z<7$. The latter is about an order of magnitude less than the former, clear evidence of a turnover.

This turnover cannot be due to obscuration, as Ostriker \& Heisler (1984 $A p . J .278,1)$ had proposed for optically-selected samples. The radio emission is unaffected by dust, and if all sources are already accounted for, there are none left to be optically obscured quasars at $z>5$. It also cannot be due to misidentifications - the high positional accuracy and the observed surface density of the identified objects rule out the possibility that all of the $z>5$ quasars are misidentified with foreground objects. Can it be argued that this turnover applies to all QSOs, and not just to radio-loud QSOs? The fact that the radio and optical QSO populations appear to turn over together seems unlikely to be a coincidence. If the optical turnover were entirely due to obscuration, then radio QSOs would also be affected, and it would be unlikely for us to have found PKS 1251-407 rather than a blank field. And the UV background also seems to exhibit a similar peak in redshift, suggestive of an epoch of activity.

We conclude that there is a real turnover in the space density of quasars at high redshift. In the context of this meeting it is interesting to note that, just as radio sources first established the strong increase in space density with increasing redshift, they are also required to confirm the decrease beyond the "quasar epoch". 